Article

\title{
Thermoelasticity of Initially Stressed Bodies with Voids: A Domain of Influence
}

\author{
Marin Marin 1,*(D), Mohamed I. A. Othman ${ }^{2}$, Sorin Vlase ${ }^{3}$ and Lavinia Codarcea-Munteanu ${ }^{1}$ \\ 1 Department of Mathematics and Computer Science, Transilvania University of Brasov, \\ 500093 Brasov, Romania; codarcealavinia@unitbv.ro \\ 2 Department of Mathematics, Faculty of Science, Zagazig University, P.O. Box 44519 Zagazig, Egypt; \\ m_i_a_othman@yahoo.com \\ 3 Department of Mechanical Engineering, Transilvania University of Brasov, 500096 Brasov, Romania; \\ svlase@unitbv.ro \\ * Correspondence: m.marin@unitbv.ro
}

Received: 9 April 2019 ; Accepted: 18 April 2019; Published: 19 April 2019

\begin{abstract}
In our study, we will extend the domain of influence in order to cover the thermoelasticity of initially stressed bodies with voids. In what follows, we prove that, for a finite time $t>0$, the displacement field $u_{i}$, the dipolar displacement field $\varphi_{j k}$, the temperature $\theta$ and the change in volume fraction $\phi$ generate no disturbance outside a bounded domain $B$.
\end{abstract}

Keywords: initially stressed bodies; dipolar structure; voids; volume fraction; domain of influence

\section{Introduction}

We must outline that our bodies are included in the thermoelasticity of bodies with voids. As we know, Nunziato and Cowin, in the paper [1], have initiated the approach of the bodies with vacuous pores (or voids). In this theory, the authors introduce an additional degree of freedom in order to develop the mechanical behavior of a body in which the skeletal material is elastic and interstices are voids of material. There are many modern applications of this theory, of which we just mention the manufactured porous materials and the geological materials like soils and rocks. In the linear case, this theory of bodies with pores was approached by Cowin and Nunziato in the study [2]. In this paper, the authors demonstrated a result of the uniqueness regarding the solution for the mixed problem and obtained a result of weak stability for the respective solutions. See also [3]. The equations of the thermoelasticity of bodies with pores were obtained by Iesan in work [4]. Other results regarding the contributions of voids of material in the theory of micropolar body with pores can be found in the papers $[5,6]$. Lately, the number of papers devoted to various aspects of microstructure has greatly increased (see [7-23], so that our work can be considered a continuation in this respect. In our present study, we extend the previous results in order to cover the thermoelasticity of initially stressed material with voids. Thus, after we put down the main equations, the initial conditions and the boundary data of the mixed value problem in this context, we define the so-called domain of influence, denoted by $B_{t}$, which corresponds to the data at time $t$ and it is associated with the mixed problem. As in previous studies (see for instance $[5,24,25]$ ), we will use a specific method in order to prove a theorem regarding the domain of influence.

Our basic result asserts that the solutions of the mixed initial-boundary value problem, in the context of the theory of thermoelasticity of bodies with voids, decay to zero outside $B_{t}$, for any finite variable $t>0$. 


\section{Basic Equations}

In our paper, we will consider some elastic bodies. We suppose that such anisotropic body is situated in regular domain $B$, include in $R^{3}$, that is, the three-dimensional Euclidian space. The border of this domain is a smooth surface, denoted by $\partial B$. The closure of $B$ is usual denoted by $\bar{B}$.

A fix system of Cartesian axes $O x_{i},(i=1,2,3)$ is used and the Cartesian notation is used for vectors and tensors. For the time derivative of a function, it is used a superposed dot. For the partial derivatives of a function with respect its spatial variables, we will use a comma which is followed by a subscript. partial derivatives with respect to the spatial coordinates. In the case of the repeated indices, the Einstein summation rule is used.

In addition, if there is no possibility of confusion, then dependence of function with regards to its spatial or time variables will be omitted. The evolution of body with dipolar structure will be described with the help of the following specific variables:

$$
u_{i}(x, t), \varphi_{i j}(x, t),(x, t) \in B \times\left[0, t_{0}\right) .
$$

Here, we denoted by $u_{i}$ the components of the displacement vector field and by $\varphi_{i j}$ the components of the dipolar displacement tensor field.

Using the above variables $u_{i}(x, t), \varphi_{i j}(x, t)$, we will introduce the components of the tensors of strain, namely $\varepsilon_{i j}, k_{i j}$ and $\gamma_{i}$, as follows:

$$
2 \varepsilon_{i j}=u_{j, i}+u_{i, j}, \gamma_{i j}=u_{j, i}-\varphi_{i j}, \chi_{i j k}=\varphi_{i j, k} .
$$

Being in the context of linear theory, it is natural to consider that internal energy density is a quadratic form with the following expression:

$$
\begin{gathered}
\varrho_{0} e=\frac{1}{2} A_{i j m n} \varepsilon_{i j} \varepsilon_{m n}+G_{i j m n} \varepsilon_{i j} \gamma_{m n}+F_{i j m n r} \varepsilon_{i j} \chi_{m n r} \\
+\frac{1}{2} B_{i j m n} \gamma_{i j} \gamma_{m n}+D_{i j m n r} \gamma_{i j} \chi_{m n r}+\frac{1}{2} C_{i j k m n r} \chi_{i j k} \chi_{m n r}+ \\
+u_{j, k} P_{k i} \varepsilon_{i j}-\varphi_{j k} Q_{k i} \gamma_{i j}+u_{j, r} N_{i r k} \chi_{i j k}+ \\
+d_{i j m} \varepsilon_{i j} \phi_{, m}+e_{i j m} \gamma_{i j} \phi_{, m}+f_{i j k m} \chi_{i j k} \phi_{, m}+ \\
+a_{i j} \varepsilon_{i j} \phi+b_{i j} \gamma_{i j} \phi+c_{i j k} \chi_{i j k} \phi+\frac{1}{2} A_{i j} \phi_{, i} \phi_{, j}- \\
+d_{i} \phi \phi_{, i}+a_{i} \theta \phi_{, i}+\xi \phi^{2}-m \theta \phi+\frac{1}{2} a \theta^{2}+ \\
-\alpha_{i j} \varepsilon_{i j} \theta-\beta_{i j} \gamma_{i j} \theta-\delta_{i j k} \chi_{i j k} \theta+\frac{1}{2} k_{i j} \theta_{, i} \theta_{, j} .
\end{gathered}
$$

We will use a procedure similar to that used by Nunziato and Cowin in [3]. Thus, taking into account that

$$
\begin{aligned}
& \tau_{i j}=\frac{\partial e}{\partial \varepsilon_{i j}}, \eta_{i j}=\frac{\partial e}{\partial \gamma_{i j}}, \mu_{i j k}=\frac{\partial e}{\partial \chi_{i j k}}, \\
& h_{i}=\frac{\partial e}{\partial \phi, i}, g=-\frac{\partial e}{\partial \phi}, \eta=\frac{\partial e}{\partial \theta}, q_{i}=\frac{\partial e}{\partial \theta_{, i}},
\end{aligned}
$$


we obtain the following constitutive equations

$$
\begin{gathered}
\tau_{i j}=u_{j, k} P_{k i}+A_{i j m n} \varepsilon_{m n}+G_{i j m n} \gamma_{m n}+F_{m n r i j} \chi_{m n r}+ \\
+a_{i j} \phi+D_{i j k} \phi_{, k}-\alpha_{i j} \theta, \\
\eta_{i j}=-\varphi_{j k} Q_{i k}+\varphi_{j k, r} N_{r i k}+G_{i j m n} \varepsilon_{m n}+B_{i j m n} \gamma_{m n}+ \\
+D_{i j m n r} \chi_{m n r}+b_{i j} \phi+e_{i j k} \phi_{, k}-\beta_{i j} \theta, \\
\mu_{i j k}=u_{j, r} N_{r i k}+F_{i j k m n} \varepsilon_{m n}+D_{m n i j k} \gamma_{m n}+C_{i j k m n r} \chi_{m n r}+ \\
+c_{i j k} \phi+f_{i j k m} \phi_{, m}-\delta_{i j k} \theta, \\
h_{i}=d_{m n i} \varepsilon_{m n}+e_{m n i} \gamma_{m n}+f_{m n r i} \chi_{m n r}+d_{i} \phi+A_{i j} \phi_{, j}-a_{i} \theta, \\
g=-a_{i j} \varepsilon_{i j}-b_{i j} \gamma_{i j}-c_{i j k} \chi_{i j k}-\xi \phi-d_{i} \phi_{, i}+m \theta, \\
\eta=\alpha_{i j} \varepsilon_{i j}+\beta_{i j} \gamma_{i j}+\delta_{i j k} \chi_{i j k}-m \phi+a_{i} \phi_{, i}+a \theta, \\
q_{i}=k_{i j} \theta_{, j} .
\end{gathered}
$$

The main equations that govern the thermoelasticity of initially stressed materials with pores are (see [5]):

- the motion equations:

$$
\begin{aligned}
& \left(\tau_{i j}+\eta_{i j}\right)_{, j}+\varrho f_{i}=\varrho \ddot{u}_{i}, \\
& \mu_{i j k, i}+\eta_{j k}+u_{j, i} Q_{i k}+\varphi_{k i} Q_{j i}- \\
& \quad-\varphi_{k r, i} N_{i j r}+\varrho g_{j k}=I_{k r} \ddot{\varphi}_{j r} ;
\end{aligned}
$$

- the equation for the equilibrium of the forces:

$$
h_{i, i}+g+\varrho l=\varrho \kappa \ddot{\phi},
$$

- the equation of energy:

$$
\varrho T_{0} \dot{\eta}=q_{i, i}+\varrho r .
$$

We complete the above equations with:

- the kinetic relations

$$
\begin{aligned}
& \varepsilon_{i j}=\frac{1}{2}\left(u_{j, i}+u_{i, j}\right), \gamma_{i j}=u_{j, i}-\varphi_{i j}, \\
& \chi_{i j k}=\varphi_{j k, i}, \theta=T-T_{0}, \phi=\varphi-\varphi_{0} .
\end{aligned}
$$

The meaning of the notations that we used in Equations (3)-(8) is as follows: $Q$ - the density of mass, which is a constant; $\eta$ - the entropy per unit mass; $T_{0}$-the temperature of the material in its undeformed state; $I_{i j}$-the tensor of microinertia; $\kappa$ - the variable inertia the equilibrated forces; $u_{i}$-the vector of the vector of moving; $\varphi_{j k}$-the tensor for dipolar moving; $\varphi$-the function for the volume fraction that in the undeformed state has the value $\varphi_{0} ; \phi$-a measure for change in volume, regarding the undeformed state; $\theta$-the variation of the temperature, regarding the temperature $T_{0}$ from the reference state; $\varepsilon_{i j}, \gamma_{i j}, \chi_{i j k}$-the strain tensors; $\tau_{i j}, \eta_{i j}, \mu_{i j k}$-the the stress tensors; $h_{i}$-the components of the vector for equilibrated stress; $q_{i}$-the vector for the flux of the heat; $f_{i}$ - the body forces; $g_{j k}$-the dipolar charges; $r$ - the measure of the supply heat; $g$ - a measure for balancing of 
intrinsic force; $L$ - a measure for balancing of extrinsic force; $A_{i j m n}, B_{i j m n}, \ldots, k_{i j}$-the functions what characterize the elastic properties of the material. These satisfy the following symmetry relations:

$$
\begin{gathered}
A_{i j m n}=A_{m n i j}=A_{j i m n}, B_{i j m n}=B_{m n i j}, a_{i j}=a_{j i}, \\
d_{i j k}=d_{j i k}, g_{i j}=g_{j i}, C_{i j k m n r}=C_{m n r i j k}, F_{i j k m n}=F_{i j k n m}, \\
G_{i j m n}=G_{i j n m}, P_{i j}=P_{j i}, k_{i j}=k_{j i} .
\end{gathered}
$$

In the above relations (1) and (3), the quantities $P_{i j}, Q_{i j}$ and $N_{i j k}$ are prescribed functions which satisfy the following equations:

$$
\left(P_{i j}+Q_{i j}\right)_{, j}=0, N_{i j k, i}+Q_{j k}=0 .
$$

Based on the inequality of the entropy production, we can deduce that

$$
k_{i j} \theta_{, i} \theta_{, j} \geq 0
$$

In order to complete our mixed initial-boundary value problem, we add to the basic Equations (2)-(7), the following prescribed initial data

$$
\begin{gathered}
u_{i}(x, 0)=u_{i}^{0}(x), \quad \dot{u}_{i}(x, 0)=u_{i}^{1}(x), \quad \varphi_{j k}(x, 0)=\varphi_{j k}^{0}(x), \quad \dot{\varphi}_{j k}(x, 0)=\varphi_{j k}^{1}(x) \\
\theta(x, 0)=\theta^{0}(x), \quad \phi(x, 0)=\phi^{0}(x), \dot{\phi}(x, 0)=\phi^{1}(x), \quad x \in \bar{B} .
\end{gathered}
$$

We also consider the given conditions to the limit

$$
\begin{aligned}
& u_{i}=\bar{u}_{i} \text { on } \partial B_{1} \times\left[0, t_{0}\right), t_{i} \equiv\left(\tau_{i j}+\eta_{i j}\right) n_{j}=\bar{t}_{i} \text { on } \partial B_{1}^{c} \times\left[0, t_{0}\right), \\
& \varphi_{j k}=\bar{\varphi}_{j k} \text { on } \partial B_{2} \times\left[0, t_{0}\right), m_{j k} \equiv \mu_{i j k} n_{i}=\bar{m}_{j k} \text { on } \partial B_{2}^{c} \times\left[0, t_{0}\right), \\
& \phi=\bar{\phi} \text { on } \partial B_{3} \times\left[0, t_{0}\right), h \equiv h_{i} n_{i}=\bar{h} \text { on } \partial B_{3}^{c} \times\left[0, t_{0}\right), \\
& \theta=\bar{\theta} \text { on } \partial B_{4} \times\left[0, t_{0}\right), q \equiv q_{i} n_{i}=\bar{q} \text { on } \partial B_{4}^{c} \times\left[0, t_{0}\right) .
\end{aligned}
$$

$n=\left(n_{i}\right)$ is the unit normal to the surface $\partial B$, outward oriented. In addition, we denoted by $\partial B_{1}, \partial B_{2}, \partial B_{3}$ and $\partial B_{4}$ the subsets of $\partial B$, considered together with their corresponding complements $\partial B_{1}^{c}, \partial B_{2}^{c}, \partial B_{3}^{c}$ and $\partial B_{4}^{c}$. The time $t_{0}$ can be infinite. The functions $u_{i}^{0}, u_{i}^{1}, \varphi_{j k}^{0} \varphi_{j k}^{1}, \theta^{0}, \phi^{0}, \phi^{1}, \bar{u}_{i}, \bar{t}_{i}, \bar{\varphi}_{j k}, \bar{\mu}_{j k}, \bar{\phi}, \bar{\theta}, \bar{q}$, $\bar{h}$ are prescribed and regular in all points where are defined.

By introducing the geometric Equation (7) and the constitutive Equation (6) into Equations (2), (3) and (5), we obtain the following system of equations:

$$
\begin{gathered}
\varrho \ddot{u}_{i}=\left[u_{j, k} P_{k i}-\varphi_{j k, r} N_{r i k}+\left(A_{i j m n}+G_{i j m n}\right) \varepsilon_{m n}+\left(G_{m n i j}+B_{i j m n}\right) \gamma_{m n}+\right. \\
\left.\left.+\left(F_{m n r i j}+D_{i j m n r}\right) \chi_{m n r}+\left(a_{i j}+b_{i j}\right) \phi+\left(d_{i j k}+e_{i j k}\right) \phi_{, k}-\left(\alpha_{i j}+\beta_{i j}\right) \theta\right)\right]_{, j}+\varrho f_{i}, \\
I_{k r} \ddot{\varphi}_{j r}=\left(u_{j, r} N_{i r k}+F_{i j k m n} \varepsilon_{m n}+D_{m n i j k} \gamma_{m n}+C_{i j k m n r} \chi_{m n r}+c_{i j k} \phi+f_{i j k m} \phi_{, m}-\delta_{i j k} \theta\right)_{, i} \\
-\varphi_{j i} Q_{k i}+\varphi_{j i, r} N_{r k i}+G_{j k m n} \varepsilon_{m n}+B_{j k m n} \gamma_{m n}+D_{j k m n r} \chi_{m n r}+b_{j k} \phi+e_{j k i} \phi_{, i}-\beta_{j k} \theta+\varrho g_{j k},
\end{gathered}
$$


We can define the solution of our mixed initial boundary value problem in the context of the theory of thermoelasticity of initially stressed bodies with voids in the cylinder $\Omega_{0}=B \times\left[0, t_{0}\right)$ as being the array $\left(u_{i}, \varphi_{j k}, \theta, \phi\right)$ that verify all equations of the system (14)-(15) for any $(x, t) \in \Omega_{0}$, and satisfy the initial data (12) and also the conditions to the limit (13).

\section{Main Result}

First of all, we start by introducing the definition of the concept of a domain of influence. Then, we will prove an inequality which will underpin the influence theorem. This inequality is a counterpart of that demonstrated in the study [5]. The main result of our work is a theorem regarding the existence of a domain of influence in the context of thermoelasticity of porous materials.

In order to obtain our results, we need to impose the following hypotheses on the properties of the bodies:

(i) $\varrho>0, \kappa>0, I_{i j}>0, T_{0}>0, a>0$;

(ii)

$$
\begin{array}{r}
A_{i j m n} x_{i j} x_{m n}+2 G_{i j m n} x_{i j} y_{m n}+B_{i j m n} y_{i j} y_{m n}+2 F_{m n r i j} x_{i j} z_{m n r}+ \\
+2 D_{i j m n r} y_{i j} z_{m n r}+C_{i j k m n r} z_{i j k} z_{m n r}+P_{k i} x_{j k} x_{j i}-2 Q_{i k} x_{j i} y_{j k}+ \\
+N_{r i k} x_{j i} z_{j k r}+2 a_{i j} x_{i j} \omega+2 b_{i j} y_{i j} \omega+2 c_{i j k} z_{i j k} \omega+2 d_{i j k} x_{i j} \omega_{k}+ \\
+2 e_{i j k} y_{i j} \omega_{k}+2 f_{i j k m} z_{i j} \omega_{m}+2 d_{i} \omega_{i} \omega+\xi \omega^{2}+A_{i j} \omega_{i} \omega_{j} \geq \\
\geq \alpha\left(x_{i j} x_{i j}+y_{i j} y_{i j}+z_{i j k} z_{i j k}+\omega_{i} \omega_{i}+\omega^{2}\right),
\end{array}
$$

for all $x_{i j}=x_{j i}, y_{j i}, z_{i j k}, \omega_{i}, \omega$;

(iii) $k_{i j} \eta_{i} \eta_{j} \geq \gamma \eta_{i} \eta_{i}$, for all $\eta_{i}$.

These hypotheses are not considered as very restrictive, as they are commonly imposed in mechanics of continuum media. As an example, the hypothesis iii is deduced from the corollary (9) and this can be obtained from the entropy production inequality.

By analogy with the step function of Heaviside, we will consider a smooth non-decreasing function $\mathcal{U}_{e}(z)$ as follows:

$$
\mathcal{U}_{e}(z)=\left\{\begin{array}{l}
0, \text { if } z \in(-\infty, 0] \\
1, \text { if } z \in[\alpha, \infty)
\end{array}\right.
$$

for a sufficiently small $e>0$.

We now fix two constants $R>0$ and $t>0$ and use $d=\left|x-x_{0}\right|$, in order to define, with the help of the above function $\mathcal{U}_{e}$, the following useful function

$$
V: B \times[0, t] \rightarrow R, \quad V(x, s)=\mathcal{U}_{e}\left(\frac{R-d}{v}+t-s\right) .
$$

$x_{0}$ is an arbitrary point fixed in $B$. Here, $v>0$ is a constant which have the amplitude of speed, which will be determined later.

Using a sphere $S\left(x_{0}, \mathcal{R}\right)$ of the form

$$
S\left(x_{0}, \mathcal{R}\right)=\left\{x \in R^{3}:\left|x-x_{0}\right|<R\right\},
$$

we define the set $\mathcal{A}$ by

$$
\mathcal{A}=\bigcup_{\alpha \in[0, t]} S\left[x_{0}, R+v(t-\alpha)\right]
$$


It is easy to see that $V(x, s)$ is a regular function in all points of $B \times[0, t]$, which decay to zero outside set $\mathcal{A}$.

We now prove an inequality which is useful in what follows.

Proposition 1. If the ordered array $\left(u_{i}, \varphi_{i j}, \phi, \theta\right)$ satisfies the system of Equations (14) and (15) and verifies Equations (12) and (13), then the following inequality takes place:

$$
\begin{gathered}
{\left[\varrho \dot{u}_{i} \dot{u}_{i}+I_{k r} \dot{\varphi}_{j r} \dot{\varphi}_{j k}+\varrho \kappa \dot{\phi}^{2}+a \theta^{2}+A_{i j m n} \varepsilon_{i j} \varepsilon_{m n}+\right.} \\
+2 G_{i j m n} \varepsilon_{i j} \gamma_{m n}+B_{i j m n} \gamma_{i j} \gamma_{m n}+2 F_{m n r i j} \varepsilon_{i j} \chi_{m n r}+ \\
+2 D_{i j m n r} \gamma_{i j} \chi_{m n r}+C_{i j k m n r} \chi_{i j k} \chi_{m n r}+2 a_{i j} \varepsilon_{i j} \phi+ \\
+2 b_{i j} \gamma_{i j} \phi+2 c_{i j k} \chi_{i j k} \phi+2 d_{i j k} \varepsilon_{i j} \phi_{, k}+2 e_{i j k} \gamma_{i j} \phi_{, k}+ \\
\left.+2 f_{i j k m} \chi_{i j k} \phi_{, k}+2 d_{i} \phi \phi_{, i}+2 A_{i j} \phi_{, i} \phi_{, j}+\xi \phi^{2}\right] \geq \\
\geq\left[\varrho \dot{u}_{i} \dot{u}_{i}+I_{k r} \dot{\varphi}_{j r} \dot{\varphi}_{j k}+\varrho \kappa \dot{\phi}^{2}+a \theta^{2}+\right. \\
\left.+\varepsilon_{i j} \varepsilon_{i j}+\gamma_{i j} \gamma_{i j}+\chi_{i j k} \chi_{i j k}+\phi^{2}+\phi_{, i} \phi_{, i}\right]
\end{gathered}
$$

for all $(x, s) \in B \times[0, t]$.

Proof. This result can be immediately deduced by taking into account the above assumptions $i$ and ii.

Let us define a function $P(x, s)$ by

$$
\begin{gathered}
P=\frac{1}{2}\left[\varrho \dot{u}_{i} \dot{u}_{i}+I_{k r} \dot{\varphi}_{j r} \dot{\varphi}_{j k}+\varrho \kappa \dot{\phi}^{2}+a \theta^{2}+A_{i j m n} \varepsilon_{i j} \varepsilon_{m n}+\right. \\
+2 G_{i j m n} \varepsilon_{i j} \gamma_{m n}+B_{i j m n} \gamma_{i j} \gamma_{m n}+2 F_{m n r i j} \varepsilon_{i j} \chi_{m n r}+ \\
+2 D_{i j m n r} \gamma_{i j} \chi_{m n r}+C_{i j k m n r} \chi_{i j k} \chi_{m n r}+P_{k i} u_{j, k} u_{j, i}- \\
-2 Q_{i k} u_{j, i} \varphi_{j k}+2 N_{r i k} u_{j, i} \varphi_{j k, r}+2 a_{i j} \varepsilon_{i j} \phi+2 b_{i j} \gamma_{i j} \phi+ \\
+2 c_{i j k} \chi_{i j k} \phi+2 d_{i j k} \varepsilon_{i j} \phi_{, k}+2 e_{i j k} \gamma_{i j} \phi_{, k}+ \\
\left.+2 f_{i j k m} \chi_{i j k} \phi_{, k}+2 d_{i} \phi \phi_{, i}+A_{i j} \phi_{, i} \phi_{, j}+\xi \phi^{2}\right] .
\end{gathered}
$$

From definition (20), we can deduce that $P$, as a function of $(t, s)$ is, in fact, the potential density energy.

In the following, we also use the function $K(x, s)$ defined by

$$
\begin{aligned}
K=\frac{1}{2}\left[\varrho \dot{u}_{i} \dot{u}_{i}+I_{k r} \dot{\varphi}_{j r} \dot{\varphi}_{j k}+\varrho \kappa \dot{\phi}^{2}+a \theta^{2}+\right. \\
\left.+\varepsilon_{i j} \varepsilon_{i j}+\gamma_{i j} \gamma_{i j}+\chi_{i j k} \chi_{i j k}+\phi^{2}+\phi, i \phi_{i}\right] .
\end{aligned}
$$

Clearly, this function is kinetic energy.

If we take into account the hypotheses $i$ and $i i$, from Equations (20) and (21), we are led to the conclusion

$$
P(x, \tau) \geq K(x, \tau), \forall(x, \tau) \in B \times[0, t] .
$$

In the following theorem, we will prove an inequality, which is helpful to obtain our main result. 
Theorem 1. If $\left(u_{i}, \varphi_{i j}, \theta, \phi\right)$ is a solution of the system of Equations (14) and (15) that verifies (12) and (13), then the next inequality is satisfied for any $(x, \tau)$ in the cylinder $B \times[0, t]$ :

$$
\begin{gathered}
\int_{D\left[x_{0}, R\right]} P(x, t) d v+\frac{1}{T_{0}} \int_{0}^{t} \int_{D\left[x_{0}, R+c(t-s)\right]} k_{i j} \theta_{i,} \theta_{, j} d v \leq \int_{D\left[x_{0}, R+c t\right]} P(x, 0) d v+ \\
+\int_{0}^{t} \int_{D\left[x_{0}, R+c(t-s)\right]} \varrho\left[f_{i} \dot{u}_{i}+g_{j k} \dot{\varphi}_{j k}+l \dot{\phi}+\frac{1}{T_{0}} r \theta\right] d v d s+ \\
\quad+\int_{0}^{t} \int_{\partial D\left[x_{0}, R+c(t-s)\right]}\left[\bar{t}_{i} \dot{u}_{i}+\bar{\mu}_{j k} \dot{\varphi}_{j k}+\bar{h} \dot{\phi}+\frac{1}{T_{0}} \bar{q} \theta\right] d S d s,
\end{gathered}
$$

for any $R>0, t>0$ and $x_{0} \in B$.

Here, $D\left(x_{0}, R\right)=\left\{\alpha \in B:\left|\alpha-x_{0}\right|<R\right\}, \partial D\left(x_{0}, R\right)=\left\{\alpha \in \partial B:\left|\alpha-x_{0}\right|<R\right\}$.

Proof. If we multiply the both members of Equation $(14)_{1}$ by $V \dot{u}_{i}$, we obtain

$$
\begin{aligned}
& \frac{1}{2} V \frac{d}{d t}\left(\varrho \dot{u}_{i} \dot{u}_{i}\right)=\varrho V f_{i} \dot{u}_{i}+\left[V\left(\tau_{i j}+\eta_{i j}\right) \dot{u}_{i}\right]_{, j}-V_{, j}\left(\tau_{i j}+\eta_{i j}\right) \dot{u}_{i}- \\
& -V\left(A_{i j m n} \varepsilon_{m n}+G_{i j m n} \gamma_{m n}+F_{i j m n r} \chi_{m n}+a_{i j} \phi+d_{i j k} \phi, k-\alpha_{i j} \theta\right) \dot{u}_{i, j} .
\end{aligned}
$$

Analogously, we multiply both sides of Equation $(14)_{2}$ by $V \dot{\varphi}_{j k}$, so that we get the equality

$$
\begin{gathered}
\frac{1}{2} V \frac{d}{d t}\left(I_{k r} \dot{\varphi}_{j r} \dot{\varphi}_{j k}\right)=\varrho V m_{j k} \dot{\varphi}_{j k}+\left(V \mu_{i j k} \dot{\varphi}_{j k}\right)_{, i}-V_{, i} \mu_{i j k} \dot{\varphi}_{j k}- \\
-V\left(F_{i j k m n} \varepsilon_{m n}+D_{m n i j k} \gamma_{m n}+C_{i j k m n r} \chi_{m n r}+c_{i j k} \phi+f_{i j k m} \phi_{, m}-\delta_{i j k}\right) \dot{\varphi}_{j k, i} .
\end{gathered}
$$

Furthermore, multiplying both sides of $(14)_{3}$ by $V \dot{\phi}$, we obtain the identity

$$
\begin{gathered}
\frac{1}{2} V \frac{d}{d t}\left(\varrho \kappa \dot{\phi}^{2}\right)=\varrho V l \dot{\phi}+\left(V h_{i} \dot{\phi}\right)_{, i}-V_{, i} h_{i} \dot{\phi}- \\
-V\left(A_{i j} \phi_{, j} \dot{\phi}_{, i}+d_{m n i} \varepsilon_{m n} \dot{\phi}_{, i}+e_{m n i} \gamma_{m n} \dot{\phi}_{, i}+f_{m n r i} \chi_{m n r} \dot{\phi}_{, i}+d_{i} \phi \dot{\phi} \dot{\phi}_{, i}-a_{i} \theta \dot{\phi}_{, i}\right)- \\
-V\left(a_{i j} \varepsilon_{i j} \dot{\phi}+b_{i j} \gamma_{i j} \dot{\phi}+c_{i j k} \chi_{i j k} \dot{\phi}+\xi \phi \dot{\phi}+d_{i} \phi_{, i} \dot{\phi}-m \theta \dot{\phi}\right) .
\end{gathered}
$$

At last, by multiplying both sides of $(14)_{4}$ by $V \theta$, we will obtain

$$
\begin{aligned}
& \frac{1}{2} G \frac{d}{d t}\left(a \theta^{2}\right)=\frac{1}{T_{0}} G r \theta+\frac{1}{\varrho T_{0}}\left[\left(G \theta q_{i}\right)_{, i}-G_{, i} \theta q_{i}\right]- \\
& -\frac{1}{\varrho T_{0}} G k_{i j} \theta_{, i} \theta_{, j}-G\left(\alpha_{i j} \theta \dot{\varepsilon}_{i j}+\beta_{i j} \theta \dot{\gamma}_{i j}+\delta_{i j k} \theta \dot{\chi}_{i j}+m \theta \dot{\phi}+a_{i} \theta \dot{\phi}_{, i}\right) .
\end{aligned}
$$


Now, by summing up, term by term, Equations (24)-(27), it is easy to deduce the identity

$$
\begin{gathered}
\frac{1}{2} V \frac{d}{d t}\left(\varrho \dot{u}_{i} \dot{u}_{i}+I_{k r} \dot{\varphi}_{j r} \dot{\varphi}_{j k}+\varrho \kappa \dot{\phi}^{2}+a \theta^{2}\right)= \\
=\varrho V f_{i} \dot{u}_{i}+\varrho V g_{j k} \dot{\varphi}_{j k}+\varrho V l \dot{\phi}+\frac{1}{T_{0}} V r \theta+ \\
+V\left[\left(\tau_{i j}+\eta_{i j}\right) \dot{u}_{j}+\mu_{i j k} \dot{\varphi}_{j k}+h_{i} \dot{\phi}+\frac{1}{\varrho T_{0}} \theta q_{i}\right]_{, i} \\
-V\left[A_{i j m n} \varepsilon_{m n} \dot{\varepsilon}_{i j}+G_{i j m n}\left(\varepsilon_{m n} \dot{\gamma}_{i j}+\dot{\varepsilon}_{m n} \gamma_{i j}\right)+\right. \\
+B_{i j m n} \gamma_{m n} \dot{\gamma}_{i j}+F_{m n r i j}\left(\varepsilon_{i j} \dot{\chi}_{m n r}+\dot{\varepsilon}_{i j} \chi_{m n r}\right)+ \\
+D_{i j m n r}\left(\gamma_{i j} \dot{\chi}_{m n r}+\dot{\gamma}_{i j} \chi_{m n r}\right)+C_{i j k m n r} \chi_{i j k} \dot{\chi}_{m n r}+ \\
+a_{i j}\left(\dot{\varepsilon}_{i j} \phi+\varepsilon_{i j} \dot{\phi}\right)+b_{i j}\left(\dot{\gamma}_{i j} \phi+\gamma_{i j} \dot{\phi}\right)+c_{i j k}\left(\dot{\chi}_{i j k} \phi+\chi_{i j k} \dot{\phi}\right)+ \\
+d_{i j k}\left(\varepsilon_{i j} \dot{\phi}_{, k}+\dot{\varepsilon}_{i j} \phi_{, k}\right)+e_{i j k}\left(\gamma_{i j} \dot{\phi}_{, k}+\dot{\gamma}_{i j} \phi_{, k}\right)+ \\
+f_{i j k m}\left(\chi_{i j k} \dot{\phi}_{, m}+\dot{\chi}_{i j k} \phi_{, m}\right)+d_{i}\left(\phi \dot{\phi}_{, i}+\dot{\phi} \phi_{, i}\right)+ \\
\left.+A_{i j} \phi_{, i} \dot{\phi}_{, j}+\xi \phi \dot{\phi}\right]-V_{, j}\left(\tau_{i j}+\eta_{i j}\right) \dot{u}_{i}-V_{, i} \mu_{i j k} \dot{\varphi}_{j k}- \\
-V_{, i} h_{i} \dot{\phi}-\frac{1}{\varrho T_{0}} V_{, i} q_{i} \theta-\frac{1}{\varrho T_{0}} V k_{i j} \theta_{, i} \theta_{, j} .
\end{gathered}
$$

It is not difficult to notice that relation (28) can be rewritten in the following equivalent form:

$$
\begin{array}{r}
\frac{1}{2} G \frac{d}{d t}\left(\varrho \dot{u}_{i} \dot{u}_{i}+I_{k r} \dot{\varphi}_{j r} \dot{\varphi}_{j k}+\varrho \kappa \dot{\phi}^{2}+a \theta^{2}+\right. \\
+A_{i j m n} \varepsilon_{m n} \varepsilon_{i j}+2 G_{i j m n} \gamma_{m n} \varepsilon_{i j}+B_{i j m n} \gamma_{m n} \gamma_{i j}+ \\
+2 F_{m n r i j} \varepsilon_{i j} \chi_{m n r}+2 D_{i j m n r} \gamma_{i j} \chi_{m n r}+C_{i j k m n r} \chi_{i j k} \chi_{m n r}+ \\
+2 a_{i j} \varepsilon_{i j} \phi+2 b_{i j} \gamma_{i j} \phi+2 c_{i j} \chi_{i j} \phi+2 d_{i j k} \varepsilon_{i j} \phi_{, k}+ \\
+2 e_{i j k} \gamma_{i j} \phi_{, k}+2 f_{i j k m} \chi_{i j k} \phi_{, m}+2 d_{i} \phi \phi_{, i}+A_{i j} \phi_{, i} \phi_{, j}+ \\
\left.+2 a_{i} \theta \phi_{, i}-2 m \theta \phi+a \theta^{2}+\xi \phi^{2}\right)= \\
=\varrho V\left(f_{i} \dot{u}_{i}+g_{j k} \dot{\varphi}_{j k}+\varrho l \dot{\phi}+\frac{1}{T_{0}} r \theta\right)+ \\
+V\left[\left(\tau_{i j}+\eta_{i j}\right) \dot{u}_{i}+\mu_{i j k} \dot{\varphi}_{j k}+h_{j} \dot{\phi}+\frac{1}{\varrho T_{0}} \theta q_{j}\right]_{, j}- \\
-V_{, j}\left(\tau_{i j}+\eta_{i j}\right) \dot{u}_{i}-V_{, i} u_{i j k} \dot{\varphi}_{j k}-V_{, i} h_{i} \dot{\phi}-V_{, i} \frac{1}{\varrho T_{0}} \theta q_{i}-\frac{1}{\varrho T_{0}} k_{i j} \theta_{, i} \theta_{, j} .
\end{array}
$$

Taking into account the expression of the potential energy $P$ from (30), identity (29) can be restated in the form

$$
\begin{gathered}
\frac{1}{2} V \dot{P}+\frac{1}{\varrho T_{0}} k_{i j} \theta_{, i} \theta_{, j}= \\
=V\left(\varrho f_{i} \dot{u}_{i}+\varrho g_{j k} \dot{\varphi}_{j k}+\varrho l \dot{\phi}+\frac{1}{T_{0}} \varrho r \theta\right)+ \\
+V\left[\left(\tau_{i j}+\eta_{i j}\right) \dot{u}_{j}+\mu_{i j k} \dot{\varphi}_{j k}+h_{i} \dot{\phi}+\frac{1}{\varrho T_{0}} \theta q_{i}\right]_{, i}- \\
-V_{, i}\left[\left(\tau_{i j}+\eta_{i j}\right) \dot{u}_{j}+\mu_{i j k} \dot{\varphi}_{j k}+h_{i} \dot{\phi}+\frac{1}{\varrho T_{0}} \theta q_{i}\right] .
\end{gathered}
$$


By integrating, over $B \times[0, t]$, both sides of identity (30) so that, by using the divergence theorem and the conditions to the limit (13), we are led to the following equality:

$$
\begin{gathered}
\int_{B} V P(x, t) d v+\frac{1}{\varrho T_{0}} \int_{0}^{t} \int_{B} V k_{i j} \theta_{, i} \theta_{, j} d v d s=\int_{B} V P(x, 0) d v+ \\
+\int_{0}^{t} \int_{\partial B} V\left(\bar{t}_{i} \dot{u}_{i}+\bar{\mu}_{j k} \dot{\varphi}_{j k}+\bar{h} \dot{\phi}+\frac{1}{\varrho T_{0}} \bar{q} \theta\right) d v d s+ \\
+\int_{0}^{t} \int_{B} \varrho V\left(f_{i} \dot{u}_{i}+g_{j k} \dot{\varphi}_{j k}+l \dot{\phi}+\frac{1}{T_{0}} r \theta\right) d v d s+ \\
+\int_{0}^{t} \int_{B} \dot{V} P(x, s) d v d s-\int_{0}^{t} \int_{B} V_{, i}\left[\left(\tau_{i j}+\eta_{i j}\right) \dot{u}_{j}+\mu_{i j k} \dot{\varphi}_{j k}+h_{i} \dot{\phi}+\frac{1}{\varrho T_{0}} q_{i} \theta\right] d v d s .
\end{gathered}
$$

Now, we consider the definition (17) of the function $V$ in order to the identity:

$$
\begin{array}{r}
\left|-V_{, j}\left(\tau_{i j}+\eta_{i j}\right) \dot{u}_{i}-V_{, i} \mu_{i j k} \dot{\varphi}_{j k}-V_{, i} h_{i} \dot{\phi}-\frac{1}{\varrho T_{0}} V_{, i} q_{i} \theta\right|= \\
=\left|\frac{1}{c} \mathcal{U}_{e}^{\prime} \frac{x_{j}}{\mathbf{r}}\left(\tau_{i j}+\eta_{i j}\right) \dot{u}_{i}+\frac{1}{c} \mathcal{U}_{e}^{\prime} \frac{x_{i}}{\mathbf{r}} \mu_{i j k} \dot{\varphi}_{j k}+\frac{1}{c} \mathcal{U}_{e}^{\prime} \frac{x_{i}}{\mathbf{r}} h_{i} \dot{\phi}+\frac{1}{c \varrho T_{0}} \mathcal{U}_{e}^{\prime} \frac{x_{i}}{\mathbf{r}} q_{i} \theta\right|= \\
=\mid \frac{1}{c} \mathcal{U}_{e}^{\prime} \frac{1}{\mathbf{r}}\left[\left(A_{i j m n} \varepsilon_{m n} x_{j}+G_{i j m n} \gamma_{m n} x_{j}+F_{i j m n r} \chi_{m n r} x_{j}+\right.\right. \\
\left.+\left(a_{i j}+b_{i j}\right) \phi x_{j}+\left(d_{i j k}+e_{i j k}\right) \phi_{, k} x_{j}-\left(\alpha_{i j}+\beta_{i j}\right) \theta x_{j}\right] \dot{u}_{i}+ \\
+\left(F_{j k m n r} \varepsilon_{m n} x_{r}+D_{j k m n r} \gamma_{m n} x_{r}+C_{i j k m n r} \chi_{m n r} x_{i}\right) \dot{\varphi}_{j k}+ \\
\left.+\left(D_{m n i} \varepsilon_{m n} x_{i}+E_{m n i} \gamma_{m n} x_{i}+A_{i j} \phi_{, j} x_{i}+d_{i} \phi x_{i}-a_{i} \theta x_{i}\right) \dot{\phi}+\frac{1}{\varrho T_{0}} k_{i j} \theta_{, j} \theta x_{i}\right] \mid,
\end{array}
$$

where we used the notation:

$$
\mathcal{U}_{e}^{\prime}=\frac{d \mathcal{U}_{e}}{d \mathbf{r}} .
$$

For the terms on the right-hand side of identity (32), we will use the arithmetic-geometric mean inequality in the form

$$
a b \leq \frac{1}{2}\left(\frac{a^{2}}{m^{2}}+b^{2} m^{2}\right)
$$

such that if we choose some suitable parameters $m$, we can find $v$ (from the definition of $\mathcal{U}_{e}$ ) to satisfy the inequality

$$
\left|-V_{, j}\left(\tau_{i j}+\eta_{i j}\right) \dot{u}_{i}-V_{, j} \mu_{i j k} \dot{\varphi}_{j k}-V_{, i} h_{i} \dot{\phi}-\frac{1}{T_{0}} V_{, i} q_{i} \theta\right| \leq \mathcal{U}_{e}^{\prime} K(x, s) .
$$

In addition, we obtain the inequality

$$
\begin{gathered}
\int_{0}^{t} \int_{B} \dot{V} P(x, s) d v d s- \\
-\int_{0}^{t} \int_{B}\left(V_{, j}\left(\tau_{i j}+\eta_{i j}\right) \dot{u}_{i}+V_{, j} \mu_{i j k} \dot{\varphi}_{j k}+V_{, i} h_{i} \dot{\phi}+\frac{1}{T_{0}} V_{, i} q_{i} \theta\right) d v d s \leq \\
\leq \int_{0}^{t} \int_{B} \mathcal{U}_{e}^{\prime}(x, \tau)[K(x, \tau)-P(x, \tau)] d v d \tau \leq 0 .
\end{gathered}
$$


Considering inequality (35), we are led to the conclusion that identity (31) receives the following form:

$$
\begin{array}{r}
\int_{B} V P(x, t) d V+\frac{1}{T_{0}} \int_{0}^{t} \int_{B} V k_{i j} \theta_{, i} \theta_{j} d v d s \leq \int_{B} V P(x, 0) d v+ \\
+\int_{0}^{t} \int_{B} \varrho V\left(f_{i} \dot{u}_{i}+g_{j k} \dot{\varphi}_{j k}+l \dot{\phi}+\frac{1}{\varrho^{2} T_{0}} r \theta\right) d v d s+ \\
+\int_{0}^{t} \int_{\partial B} V\left(\bar{t}_{i} \dot{u}_{i}+\bar{\mu}_{j k} \dot{\varphi}_{j k}+\bar{h} \dot{\phi}+\frac{1}{\varrho T_{0}} \bar{q} \theta\right) d v d s .
\end{array}
$$

Finally, if we will pass to the limit in relation (36), as $e \rightarrow 0$, then we deduce that the (boundedly) limit of the function $V$ is the indicator function, also called the characteristic function, for the set $\mathcal{A}$, defined after (18). As an immediate consequence, we are led to the inequality (23), such that Theorem 1 is concluded.

The previous estimations obtained in Theorem 1, Proposition 1 will be used to obtain the main theorem of our present work, which is a generalization of the domain of influence result.

Let us denote by $B(t)$ the set that contains the points $x$ from $\bar{B}$ such that:

(1) for $x \in B, u_{i}^{0} \neq 0$ or $u_{i}^{1} \neq 0$ or $\varphi_{j k}^{0} \neq 0$ or $\varphi_{j k}^{1} \neq 0$ or $\phi^{0} \neq 0$ or $\phi^{1} \neq 0$ or $\theta^{0} \neq 0$ or

$\exists \alpha \in[0, t]$ so that $f_{i}(x, \alpha) \neq 0$ or $m_{i}(x, \alpha) \neq 0$ or $l(x, \alpha) \neq 0$ or $r(x, \alpha) \neq 0$;

(2) for $x \in \partial B_{1}, \exists \alpha \in[0, t]$ so that $\bar{u}_{i}(x, \alpha) \neq 0$;

(3) for $x \in \partial B_{1}^{c}, \exists \alpha \in[0, t]$ so that $\bar{t}_{i}(x, \alpha) \neq 0$;

(4) for $x \in \partial B_{2}, \exists \alpha \in[0, t]$ so that $\bar{\varphi}_{j k}(x, \alpha) \neq 0$;

(5) for $x \in \partial B_{2}^{c}, \exists \alpha \in[0, t]$ so that $\bar{\mu}_{j k}(x, \alpha) \neq 0$;

(6) for $x \in \partial B_{3}, \exists \alpha \in[0, t]$ so that $\bar{\phi}(x, \alpha) \neq 0$;

(7) for $x \in \partial B_{3}^{c}, \exists \alpha \in[0, t]$ so that $\bar{h}(x, \alpha) \neq 0$;

(8) for $x \in \partial B_{4}, \exists \alpha \in[0, t]$ so that $\bar{\theta}(x, \alpha) \neq 0$;

(9) for $x \in \partial B_{4}^{c}, \exists \alpha \in[0, t]$ so that $\bar{q}(x, \alpha) \neq 0$.

At the instant $t$, the domain of influence of the data, $B_{t}$, is a set defined by

$$
B_{t}=\left\{x_{0} \in \bar{B}: B(t) \cap \bar{S}\left(x_{0}, v t\right) \neq \Phi\right\},
$$

where $\Phi$ is the notation for the empty set and the sphere $S\left(x_{0}, v t\right)$ is defined in Equation (18).

Now, we can prove the main result of our study.

Theorem 2. If the array $\left(u_{i}, \varphi_{i j}, \theta, \phi\right)$ verifies all equations of the system of Equations (14) and (15) and satisfies the conditions (12) and (13), we obtain a characterization of the solution as follows:

$$
u_{i}=0, \varphi_{i j}=0, \theta=0 \text { and } \phi=0, \text { for }(x, \tau) \in\left\{\bar{B} \backslash B_{t}\right\} \times[0, t] .
$$

Proof. We will use inequality (23) considered for an arbitrary $x_{0}, x_{0} \in \bar{B} \backslash B_{t}$ and $\tau \in[0, t]$, by taking the values $t=\tau$ and $R=v(t-\tau)$. Then, we obtain

$$
\begin{gathered}
\int_{D\left[x_{0}, v(t-\tau)\right]} P(x, \tau) d V+\frac{1}{T_{0}} \int_{0}^{\tau} \int_{D\left[x_{0}, v(t-s)\right]} k_{i j} \theta_{, i} \theta_{j} d V d s \leq \\
\leq \int_{\left.D\left[x_{0}, v t\right)\right]} P(0, x) d v+\int_{0}^{\tau} \int_{D\left[x_{0}, v(t-s)\right]} \varrho\left(f_{i} \dot{u}_{i}+g_{j k} \dot{\varphi}_{j k}+l \dot{\phi}+\frac{1}{T_{0}} r \theta\right) d V d s+ \\
+\int_{0}^{\tau} \int_{\partial D\left[x_{0}, v(t-s)\right]} \varrho\left(\bar{t}_{i} \dot{u}_{i}+\bar{\mu}_{j k} \dot{\varphi}_{j k}+\bar{h} \dot{\phi}+\frac{1}{T_{0}} \bar{q} \theta\right) d S d s .
\end{gathered}
$$


However, $x_{0} \in \bar{B} \backslash B_{t}$, so we can deduce that $x \in D\left(x_{0}, v t\right)$ and therefore $x \notin B(t)$. Thus, we are led to the conclusion that

$$
\int_{D\left[x_{0}, v t\right]} P(0, x) d v=0
$$

Taking into account that, because of $D\left[x_{0}, v(t-s)\right] \subseteq D\left(x_{0}, v t\right)$, we get

$$
\int_{0}^{\tau} \int_{D\left[x_{0}, v(t-s)\right]} \varrho\left(f_{i} \dot{u}_{i}+g_{j k} \dot{\varphi}_{j k}+l \dot{\phi}+\frac{1}{T_{0}} r \theta\right) d V d s=0
$$

and

$$
\int_{0}^{\tau} \int_{D\left[x_{0}, v(t-s)\right]}\left(\bar{t}_{i} \dot{u}_{i}+\bar{\mu}_{j k} \dot{\varphi}_{j k}+\bar{h} \dot{\phi}+\frac{1}{T_{0}} \bar{q} \theta\right) d V d s=0 .
$$

Now, considering the hypothesis iii and taking into account the relations (40)-(42), we deduce

$$
\int_{D\left[x_{0}, v(t-s)\right]} P(s, x) d v \leq 0 .
$$

The inequality (43) together with the inequality (22) lead to the conclusion

$$
\int_{D\left[x_{0}, v(t-s)\right]} K(x, s) d V \leq 0
$$

so that, considering the definition (21) of the function $K$, we obtain

$$
\dot{u}_{i}\left(x_{0}, s\right)=0, \dot{\varphi}_{j k}\left(x_{0}, s\right)=0, \theta\left(x_{0}, s\right)=0, \phi\left(x_{0}, s\right)=0
$$

for all $\left(x_{0}, s\right) \in\left\{\bar{B} \backslash B_{t}\right\} \times[0, t]$.

At last, because $u_{i}\left(x_{0}, 0\right)=0, \varphi_{j k}\left(x_{0}, 0\right)=0$ for all $x_{0} \in \bar{B} \backslash B_{t}$, we get

$$
u_{i}\left(x_{0}, s\right)=0, \varphi_{j k}\left(x_{0}, s\right)=0, \theta\left(x_{0}, s\right)=0, \phi\left(x_{0}, s\right)=0
$$

for all $\left(x_{0}, s\right) \in\left\{\bar{B} \backslash B_{t}\right\} \times[0, t]$ so that the proof of Theorem 2 is complete.

\section{Conclusions}

We want to emphasize that our main result from the present study is a generalization of the result regarding the domain of influence theorem from classical elasticity and this extension is made in a more complex context, one of the theory of thermoelastic body with dipolar structure and with voids. Thus, we have proven that the result regarding the domain of influence is still valid even if we are out of the framework of classical elasticity.

Namely, we need to emphasize that the validity of the domain of influence result was not affected by the fact that we considered the effect of thermal treatment, the effect of the dipolar structure and the effect of voids.

Author Contributions: All authors contributed equally to the writing of this paper. All authors read and approved the final form of the manuscript.

Funding: This research received no external funding

Acknowledgments: We want to thank the reviewers who have read the manuscript carefully and have proposed pertinent corrections that have led to an improvement in our manuscript.

Conflicts of Interest: The authors declare no conflict of interest. 


\section{References}

1. Nunziato, J.W.; Cowin, S.C. A nonlinear theory of materials with voids. Arch. Rat. Mech. Anal. 1979, 72, 175-201. [CrossRef]

2. Cowin, S.C Nunziato, J.W. Linear elastic materials with voids. J. Elast. 1983, 13, 125-147. [CrossRef]

3. Goodman, M.A.; Cowin, S.C. A continuum theory of granular materia. Arch. Rat. Mech. Anal. 1971, 44, 249-266. [CrossRef]

4. Iesan, D. A theory of thermoelastic material with voids. Acta Mech. 1986, 60, 67-89. [CrossRef]

5. Marin, M.; Marinescu, C. Thermoelasticity of initially stressed bodies, asymptotic equipartition of energies. Int. J. Eng. Sci. 1998, 36, 73-86. [CrossRef]

6. Marin, M. Cesaro means in thermoelasticity of dipolar bodies. Acta Mech. 1997, 122, 155-168. [CrossRef]

7. Eringen, A.C. Theory of micromorphic materials with memory. Int. J. Eng. Sci. 1972, 10 623-641. [CrossRef]

8. Eringen, A.C. Theory of thermo-microstretch elastic solids. Int. J. Eng. Sci. 1990, 28, 1291-1301. [CrossRef]

9. Mindlin, R.D. Micro-structure in linear elasticity. Arch. Ration. Mech. Anal. 1964, 16, 51-78. [CrossRef]

10. Green, A.E. Rivlin, R.S. Multipolar continuum mechanics. Arch. Ration. Mech. Anal. 1964 17, $113-147$. [CrossRef]

11. Fried, E.; Gurtin, M.E. Thermomechanics of the interface between a body and its environment. Continuum Mech. Thermodyn. 2007, 19, 253-271. [CrossRef]

12. Abbas, I.A. A GN model based upon two-temperature generalized thermoelastic theory in an unbounded medium with a spherical cavity. Appl Math Comput. 2014, 245, 108-115. [CrossRef]

13. Abbas, I.A. Eigenvalue approach for an unbounded medium with a spherical cavity based upon two-temperature generalized thermoelastic theory. J Mech. Sci. Technol. 2014, 28, 4193-4198. [CrossRef]

14. Abbas, I.A. Abo-Dahab, S.M. On the numerical solution of thermal shock problem for generalized magneto-thermoelasticity for an infinitely long annular cylinder with variable thermal conductivity. J. Comput. Theor. Nanosci. 2014, 11, 607-618. [CrossRef]

15. Othman, M.I.A. State Space Approach to Generalized Thermoelasticity Plane Waves with Two Relaxation Times under the Dependence of the Modulus of Elasticity on Reference Temperature. Can. J. Phys. 2003, 81, 1403-1418 [CrossRef]

16. Sharma, J.N.; Othman, M.I.A. Effect of Rotation on Generalized Thermo-viscoelastic Rayleigh-Lamb Waves. Int. J. Solids Struct. 2007, 44, 4243-4255. [CrossRef]

17. Othman, M.I.A. Hasona, W.M. Abd-Elaziz, E.M. Effect of Rotation on Micropolar Generalized Thermoelasticity with Two-Temperatures using a Dual-Phase-Lag Model. Can. J. Phys. 2014, 92, 149-158. [CrossRef]

18. Marin, M.; Öchsner, A. The effect of a dipolar structure on the Holder stability in Green-Naghdi thermoelasticity. Contin. Mech. Thermodyn. 2017, 29, 1365-1374. [CrossRef]

19. Hassan, M.; Marin, M.; Alsharif, A.; Ellahi, R. Convective heat transfer flow of nanofluid in a porous medium over wavy surface. Phys. Lett. A 2018, 382, 2749-2753. [CrossRef]

20. Marin, M.; Nicaise, S. Existence and stability results for thermoelastic dipolar bodies with double porosity. Contin. Mech. Thermodyn. 2016, 28, 1645-1657. [CrossRef]

21. Marin, M. Lagrange identity method for microstretch thermoelastic materials. J. Math. Anal. Appl. 2010, 363, 275-286. [CrossRef]

22. Modrea, A.; Vlase, S.; Calin, R.; Peterlicean, A. The influence of dimensional and structural shifts of the elastic constant values in cylinder fiber composites. J. Optoelectron. Adv. Mater. 2013, 15, 278-283.

23. Niculita, C.; Vlase, S.; Bencze, A.; Mihalcica, M. Optimum stacking in a multi-ply laminate used for the skin of adaptive wings. J. Optoelectron. Adv. Mater. 2011, 5, 1233-1236.

24. Carbonaro, B.; Russo, R. Energy inequalities in classical elastodynamics. J. Elast. 1984, 14, 163-174. [CrossRef]

25. Chandrasekharaiah, D.S. An uniqueness theorem in the theory of elastic. J. Elast. 1987, 18, $173-179$. [CrossRef]

(C) 2019 by the authors. Licensee MDPI, Basel, Switzerland. This article is an open access article distributed under the terms and conditions of the Creative Commons Attribution (CC BY) license (http://creativecommons.org/licenses/by/4.0/). 